DOI 10.15826/B978-5-7996-3073-7.4

Gershom T. Lazarus

Emory University,

Atlanta, GA, USA

Christine L. Ginalis

The Graduate Center, CUNY,

New York, United States

\title{
Applying principles of Luria's theory to the fetal stage and integration of psycho-physiological mechanisms to optimize adaptive behavior
}

Abstract. Luria proposed that the brain is organized hierarchically and according to functional units. The functional units provide a basis of integrated experience and behavior that becomes fractionated in various psychological and behavioral disorders. Emerging research suggests that the fundamental organization of brain networks and functional connections are established in utero during the second and third trimesters of fetal development. This paper discusses the value of studying emerging fetal organization within the framework of Luria's theory. In addition, it will expand Luria's theory in emphasizing the integration of physiological and psychological mechanisms across the life-span, with the aim of developing more individualized assessment and intervention tools.

Keywords: Life-span development, physiology, fetal brain development, Luria.

Introduction. We propose an integrated model of optimal development comprising the physiological, psychological, and neurological/ neuropsychological mechanisms associated with pathology that encompasses an intersection of the autonomic nervous system (ANS), central nervous system (CNS), immune system, and hypothalamicpituitary-adrenal (HPA) axis. Luria proposes that the brain functions as a complex network of regions that communicate with each other, known as the functional connectome [1]. Our model extends Luria's principals by including physiological components in this network that 
impact brain development and behavior. We will discuss applying this model in neuropsychological practice, from the fetal stage of development to adulthood. Heart Rate Variability (HRV) Biofeedback is an intervention that guides an individual's breathing patterns with the benefit of increasing heart rate variability to optimize physiological homeostasis [2]. The paper discusses the use of HRV biofeedback in two main areas; fetal development and psychotherapy. Data from an experimental procedure on maternal-fetal dyads and a psychotherapy case study will be used to highlight the use of the HRV Biofeedback protocol and provide evidence for including physiological assessments and intervention, when exploring brain-behavior relationships.

Materials and methods. HRV Biofeedback. The ANS power spectrum provides an indication of activation of ANS components. A single sharp peak around $0.1 \mathrm{~Hz}$ was used as an indicator of baroreceptor activation and evidence for "training" the ANS system to respond optimally to stimuli.

Fetal. Thirty-three maternal-fetal dyads participated in a HRV Biofeedback session. Fetal CNS/ANS developmental indicators (fetal movement and HR), maternal psychological (self-reported), maternal HPA axis (cortisol), and maternal ANS (HRV) indices were analyzed pre, during, and post-biofeedback intervention. The current lecture will focus on fetal movement indices.

Psychotherapy. The case study outlines a psychotherapy client diagnosed with Prader Willi and Autism Spectrum Disorder. Treatment included HRV Biofeedback in addition to "traditional" psychotherapy. Behavioral and physiological (ANS) measures were tracked across 13 sessions.

Results. Fetal. Preliminary data analyses reveal that maternal HRV biofeedback is associated with shifts in fetal movement, both decreased and increased fetal movement, when compared to a baseline period with fetal movement decrease reaching statistical significance. The data suggests that overall more fetuses fell into the "fetal movement decrease" group compared to the "fetal movement increase group."

Psychotherapy. Analysis of case study data reveals a parallel improvement in behavioral responding (parent and patient reports as well as behavioral observations during sessions) and ANS functioning (peak 
around $0.1 \mathrm{~Hz}$ ) across the sessions. By session 13, the patient described situations in which he was able to control impulsive behaviors; situations that previously resulted in a loss of control. In addition, the patient demonstrated an improved ANS response under conditions of biofeedback training from session 1 to 13 .

Conclusion. Our findings provide preliminary evidence for the use of HRV Biofeedback to stimulate fetal neuro-motor pathways. The variability noted between fetuses under conditions of biofeedback suggests that individual differences are present early in development and necessitates further investigation.

The theory underlying the use of maternal HRV biofeedback to modulate fetal movement stemmed from the research on maternal-fetal synchrony. The goal was to stimulate the fetal neuro-motor pathways with the underlying premise of increasing the range of neurobehavioral responding. Changes in fetal general movements are sub-served by the cortical subplate, and connections to the brain stem, thalamus, and cortex via corticospinal and thalamocortical tracts. The development of fetal movements parallels fetal brain development. Fetal brain development is characterized by the interaction between transient and permanent neural structures throughout the fetal gestational period. Sensory stimulation is crucial in shaping cerebral structures and functional connections instigated by genetic factors. One of the key indices of a healthy developing nervous system is "response to stimulus" and assessment of fetal general movement provides insight into fetal development.

Findings from the psychotherapy case study provides impetus to integrate physiological and behavioral tools in mental health treatment. The correlation between physiological indices and "real-world" adaptive behavioral changes supports an integrated model of optimal functioning. Particularly in individuals with altered cognitive capacities, targeting physiological mechanisms provide an alternate route to modifying behavior as compared to "traditional" psychotherapies. Luria's emphasis on the use of "structural units" in a "functional system" [3] can be utilized with increased emphasis on the integration of physiological and psychological "units" to optimize adaptive behaviors from the fetal stage of development through to the adult. 
1. Luria A. R. The Working Brain. An introduction to neuropsychology. London: Penguin books, 1973.

2. Lehrer P. M., Gevirtz R. Heart rate variability biofeedback: how and why does it work? // Frontiers in psychology. 2014. № 5. P. 756.

3. Luria A. R. Restoration of function after brain injury. New York: Pergamon, 1963. 